This is the peer reviewed version of the following article: Mendoza F, Ferrús R, Sallent O., "Experimental proof of concept of an SDN-based traffic engineering solution for hybrid satellite-terrestrial mobile backhauling", Int J Satell Commun Network 2019;1-16, which has been published in final form at https://doi.org/10.1002/sat.1303. This article may be used for non-commercial purposes in accordance with Wiley Terms and Conditions for Üse of Self-Archived Versions.

\title{
Experimental Proof of Concept of an SDN-based Traffic Engineering Solution for Hybrid Satellite-Terrestrial Mobile Backhauling
}

\author{
Fabian Mendoza $^{1}$ | Ramon Ferrus ${ }^{1}$ | Oriol Sallent ${ }^{1}$
}

\footnotetext{
${ }^{1}$ Signal Theory and Communications, Universitat Politecnica de Catalunya (UPC), Barcelona, Spain
}

\section{Correspondence}

Fabian Mendoza,

email:

jesus.fabian.mendoza@tsc.upc.edu.com Ramon Ferrus,

email: rferrus@tsc.upc.edu.com Oriol Sallent,

email: osallent@tsc.upc.edu.com

\begin{abstract}
Summary
Satellite networks are expected to be an integral part of $5 \mathrm{G}$ service deployment. One compelling use case is mobile backhauling, where the exploitation of a satellite component can improve the reach, robustness and economics of $5 \mathrm{G}$ rollout. The envisaged availability of new satellite capacity together with the development of better integration approaches for the provisioning and operation of the satellite component in a more flexible, agile and cost-effective manner than done today are expected to revamp such use case within the $5 \mathrm{G}$ ecosystem. In this context, sustained in the architectural designs proposed within H2020 VITAL research project, this paper presents an experimental Proof of Concept $(\mathrm{PoC})$ of a satellite-terrestrial integration solution that builds upon Software-Defined Networking (SDN) technologies for the realization of End-to-End Traffic Engineering (E2E TE) in mobile backhauling networks with a satellite component. A laboratory testbed has been developed and validated, consisting of a small-scale private mobile network with a backhaul setting that combines Ethernet wired links, a satellite link emulator (OpenSAND), OpenFlow switches and an OpenFlow controller running the network application for E2E TE. Provided results show the operation of a E2E TE application able to enforce different traffic routing and path failure restoration policies as well as the performance impact that it has on the mobile network connectivity services.
\end{abstract}

\section{KEYWORDS:}

Hybrid Satellite-Terrestrial Backhaul Networks, Traffic Distribution Strategies, Resilience schemes, 5G mobile networks

\section{1 | INTRODUCTION}

Higher network availability levels, ubiquitous connectivity extended to remote and rural low-density areas as well as long-haul transportation (e.g. aircraft, trains) are recognized as key requirements for $5 \mathrm{G}$ networks ${ }^{112}$. To fulfil these requirements, satellite networks are expected to be an integral part of $5 \mathrm{G}$ service deployment, contributing their unique wide-scale geographical

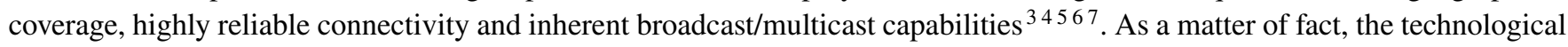
advances in the satellite domain such as the use of High Throughput Satellites (HTS) in Geostationary Earth Orbit (GEO) is currently transforming the way that satellite capacity is brought into the market. Over 100 HTS systems are espected to be in orbit by 2020-2025, delivering Tbps of connectivity in Ku- and Ka- bands at reduced $\operatorname{cost}^{8}{ }^{8}$. Likewise, a large number of 
non-GEO low-cost micro-satellites constellations are expected to come to fruition in the forthcoming years, anticipating a further capacity cost reduction and improved performance in terms of latency 9 .

Remarkably, the potential roles and benefits of satellite networks in 5G have been acknowledged within the 3rd Generation Partnership Project (3GPP) in charge of 5G system specifications. In this regard, 3GPP specifications have adopted requirements that mandate $5 \mathrm{G}$ systems to be able to provide services using satellite access as well as to support the use of high-latency satellite backhaul links between the Radio Access Network (RAN) and core network 10. Accordingly, several 3GPP study items are currently on-going to undertake the necessary technical studies for the support of satellite as well as High Altitude Platforms (HAPS) as part of next generation $5 \mathrm{G}$ systems 11 .

In this context, satellite backhaul stands out as one of the most compelling scenarios anticipated to gain further relevance in $5 \mathrm{G}^{35}$. In particular, satellite backhauling can be instrumental in providing backhaul connectivity to base stations (BSs), deployed in hard to reach areas or installed on board a transportation/moving vehicle with no other feasible backhauling means. In addition, satellite backhaul links can also bring important advantages when deployed and operated in combination with a terrestrial backhaul. In this regard, combined satellite-terrestrial backhauling solutions can increase network availability and resiliency by e.g., using the satellite backhaul as backup capacity for terrestrial link failures in critical cell sites $\frac{12}{12}$ and temporary BS deployments in special events and emergency situations 13 . Ultimately, a combined satellite-terrestrial backhauling solution can also turn into more efficient traffic delivery to BSs. For example, jointly exploited with the terrestrial capacity, a pool of satellite capacity can be used for traffic offloading and load balancing (e.g. diverting traffic from congested areas so the terrestrial capacity gets supplemented during peak-times) as well as for multicast/broadcast traffic delivery to multiple cell sites (e.g. content edge caches, live TV stream distribution) in a more resource efficient manner 14/15/16.

A full realization of a combined satellite-terrestrial backhauling scenario requires improved integration approaches for the provisioning and operation of the satellite component in a more flexible, agile and cost-effective manner than currently done. In this regard, the evolution of satellite ground segment systems (e.g. satellite gateways and terminals) from today's rather closed solutions towards more open architectures based on Software Defined Networking (SDN) and Network Function Virtualization (NFV) technologies arises as a necessary step. This evolution is not only expected to bring into the satellite domain the benefits associated with the advances in network softwarization technologies consolidating within the 5G landscape, but also to greatly facilitate the seamless integration and operation of combined satellite and terrestrial networks.

In the pursue of such a goal, important advances have been carried out in the recent years with regard to the analysis of the potential use cases, requirements and definition of functional frameworks for the exploitation of SDN and/or NFV technologies in satellite networks 17 - 25. Pioneering work in this area by Bertaux and al. ${ }^{17}$ investigated the advantages of introducing network programmability and virtualization using SDN and/or NFV through the analysis of four use cases as well as their impacts on a typical satellite system architecture. A more extended set of use cases, delineating opportunities as well as research challenges ahead, was elaborated by Ferrús at al. in 18 in the context of the European H2020 Virtualized Hybrid Satellite-Terrestrial Systems for Resilient and Flexible Future Networks (VITAL) research project 19. Considering the requirements enacted from the use cases, VITAL project delivered a feasibility study of different functional splits for the virtualization of a satellite gateway and developed a generic functional architecture for satellite ground segment systems embracing NFV and SDN technologies 19 . Moreover, the applicability of the VITAL functional architecture in a combined satellite-terrestrial backhauling scenario was further developed in 2627, with a focus on the use of SDN technologies for the realization of end-to-end Traffic Engineering (TE) applications across the terrestrial and satellite segments. The benefits of such architecture were assessed in 28 in terms of improved network resource efficiency achieved through the centralized and more fine-grained control of traffic routing enabled by the SDN-based TE applications. Other relevant research projects coping with the applicability of SDN/NFV technologies are currently on-going 2021. In this respect, an overview of the current $5 \mathrm{G}$ initiatives and projects followed by a proposed architecture for $5 \mathrm{G}$ satellite networks where the SDN/NFV approach facilitates the integration with the $5 \mathrm{G}$ terrestrial system is provided in 23 , which also analyses a novel technique based on network coding for the joint exploitation of multiple paths in integrated satellite-terrestrial systems.

Nevertheless, while important progress has been achieved so far on network architectural and functional aspects as well as on the assessment of their benefits mainly via mathematical modelling and more or less sophisticated simulation environments, 
further research is still warranted towards the practical implementation of integrated satellite-terrestrial solutions and their assessment under more realistic conditions. In this context, sustained on the outcomes delivered by the VITAL project and further progressing in this research area, this paper presents an experimental Proof of Concept (PoC) of a SDN-based integration solution for the satellite component in a hybrid satellite-terrestrial mobile backhaul network and the practical realization of SDN-based TE applications. In particular, building upon the functional architecture proposed in project VITAL, a laboratory testbed has been developed to showcase the programming and operation of SDN-based TE applications able to enforce different traffic routing and path failure restoration policies in a hybrid backhaul network. Reported results of the PoC shed light on valuable operational data of the proposed integration solution such as the execution times of the TE mechanisms for activation and deactivation of the routing paths and the incurred signaling overhead. In addition, the PoC provides an assessment of the performance impact caused by the applied TE policies on the QoS of the mobile network connectivity services.

The rest of the paper is organized as follows. Section 2 briefly outlines the main architectural traits of the satellite-terrestrial SDN-based integration solution that is used as the conceptual foundation of this work. Next, Section 3 describes the implemented SDN-enabled hybrid satellite-terrestrial mobile backhaul testbed, detailing its components and service configuration settings as well as the structure and logic of the programmed E2E TE applications. On this basis, the network operational validation and performance assessment is provided in Section 4. Finally, Section 5 draws the conclusions.

\section{2 | SDN-BASED INTEGRATION SOLUTION FOR HYBRID SATELLITE-TERRESTRIAL MOBILE BACKHAUL}

Mobile networks have been progressively embracing SDN concepts and technologies to decouple the control plane from the user plane. In this regard, the so called Control and User Plane Separation (CUPS) architecture has been developed as an enhancement of the 4G/LTE standards to fully split control and user plane functions within the Evolved Packet Core (EPC) ${ }^{29}$. Likewise, the new $5 \mathrm{G}$ Core Network $(5 \mathrm{CN})$ specifications have consolidated this separation as a key design principle 30 . An illustrative view of the architecture of a mobile network with SDN-enabled transport from the Radio Access Network (RAN) nodes (e.g. BSs) all the way through the backhaul to the core network is depicted in Fig. 1

While conceptually valid for the $5 \mathrm{G}$ system architecture, the architectural view illustrated in Fig. 1 is contextualized for 4G/LTE technology to facilitate the mapping with the developed testbed, which is built upon currently available LTE equipment for lab testing. As depicted in Fig. 1 the control functions of the Mobile Core Network (MCN) (e.g. EPC control functions) are implemented as network applications that, through northbound Application Program Interfaces (APIs) provided by a SDN controller, command the operation of the data plane of the transport network (i.e. the Network Elements [NEs] with packet switching and forwarding capabilities). In this way, such SDN controller becomes instrumental for managing the connectivity services offered by the transport network (e.g. activation / deactivation of traffic flows with specific QoS settings between specific network end-points) according to the needs of the MCN applications. Moreover, the SDN controller can also be leveraged by TE applications intended to optimize the E2E transport network performance by dynamically analyzing, predicting, and regulating the traffic behavior across the network. Of note is that the SDN controller is represented here as a single functional entity even though, in a practical realization, it is likely to follow a hierarchical structure of controllers.

Consistently with such an SDN-based mobile network architecture, the solution developed in ${ }^{2627}$ for the integration of a satellite network within the transport network relies on the exposure by the satellite network of an SDN-based control interface for the SDN controller to manage the satellite network connectivity services as it is done with the rest of NEs. This approach is illustrated in Fig. 1 by showing the interaction of the SDN controller with the satellite network control plane functions of the satellite network (e.g. Network Control Centre [NCC] functions in 31). The realization of such SDN-based interface for the satellite network could actually leverage one or a combination of different interfaces and data models gaining traction in (terrestrial) networking such as OpenFlow $(\mathrm{OF})^{32}$, Open Networking Foundation Transport API (T-API) ${ }^{33}$, ONF Microwave Information Model ${ }^{[34}$ and IETF Netconf along with YANG models specified for traffic engineered networks ${ }^{35 !}$. Among them, the OF specification is proposed in ${ }^{2627}$ to abstract the overall behaviour of the satellite network as a switch, enabling in this way a fine-grained control of the forwarding behaviour of the satellite network connectivity services through conventional flow management procedures ${ }^{36}$. Of note is that the exposure of an OF interface by a satellite network has been also proposed in the 


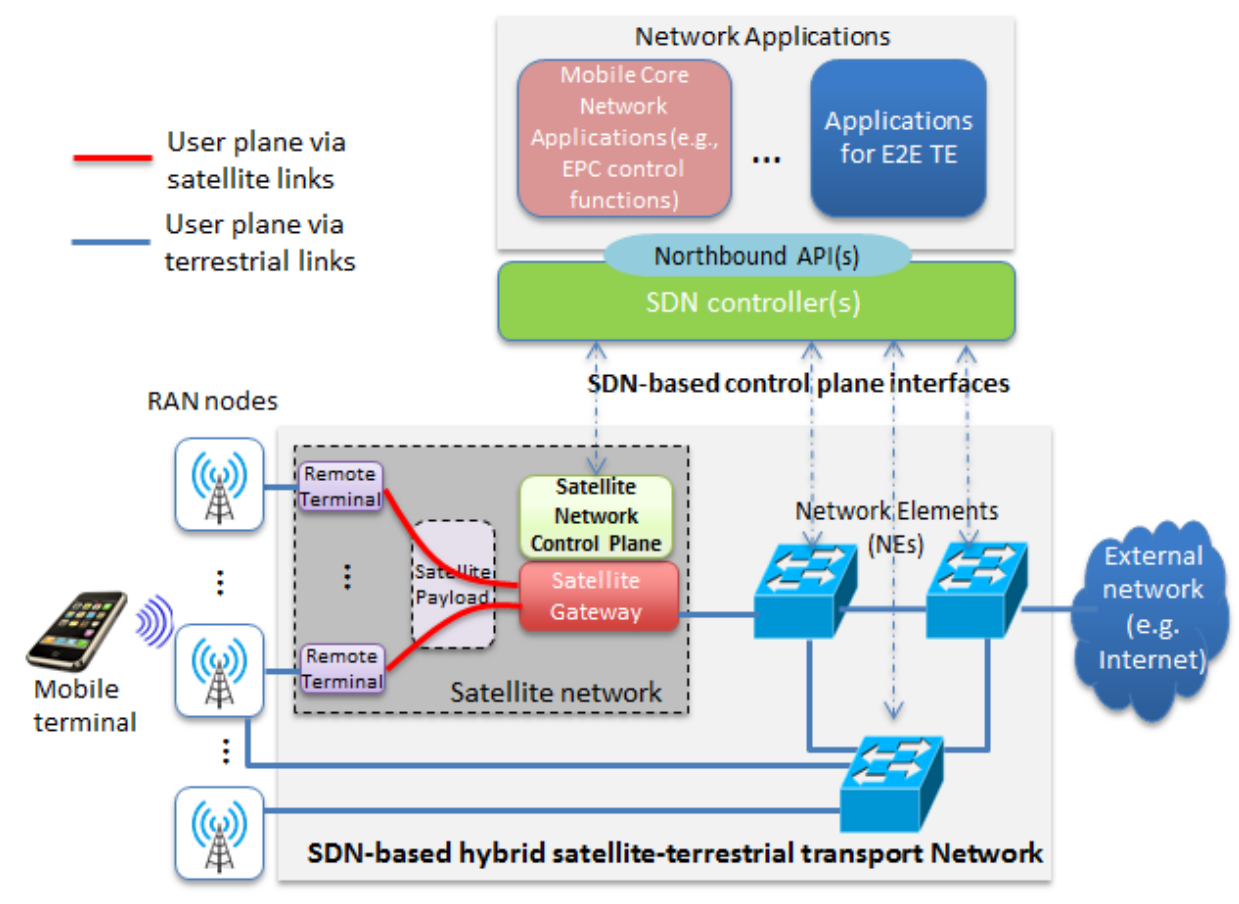

FIGURE 1 Illustrative view of a SDN-based mobile network with a satellite component for mobile backhauling within the transport network.

context of the realization of Virtual Network Operator (VNO) solutions ${ }^{32}$, in which a VNO is provided with an interface to control and manage the satellite segment resources leased from a satellite network operator as if it was programming a switch OF. For the interested reader, further insights on the functional architecture of the SDN-based satellite network, detailing the characteristics of both externally exposed and internal interfaces and including a study of the pros and cons of several interfaces and data models that could be leveraged is given in 37 .

Regarding the realization of TE mechanisms, the key advantage of the proposed integration solution compared to the traditional TE mechanisms used in today's transport networks (e.g. Multi-Protocol Label Switching TE [MPLS-TE]) stems from the SDN control centralization that provides a holistic view of the network together with the necessary mechanisms to enforce network polices from a single touch point. More specifically, in the hybrid satellite-terrestrial transport network scenario, such centralized control allows for the use of the satellite capacity in the way that best complements the terrestrial capacity in front of the changing conditions of both traffic demand (e.g. increase of traffic demand for an especial event, spatial demand fluctuations over time) and network situation (e.g. backhaul backup for terrestrial link failures, network rapid roll-out, fast response capacity, cells on wheels). Indeed, the performance analysis reported in ${ }^{28}$ shows how a given amount of satellite capacity provisioned for resilience purposes can be exploited to maximize a global network utility under both normal operation and a number of failure conditions in the terrestrial links. Such work is leveraged here for the implementation of the decision-making logic of the TE application showcased in the testbed.

\section{3 | EXPERIMENTAL TESTBED}

A high level view of the experimental testbed is depicted in Fig. 2 The testbed comprises a private, small-scale LTE network that uses a SDN-enabled hybrid satellite-terrestrial network for backhauling the traffic between a BS node (called eNB in LTE) and a MCN node with the full LTE EPC functionality. The LTE network is implemented with the AMARI OTS 100 system commercialized by Amarisoft $\frac{38}{38}$, which is executed over general-purpose Linux Personal Computers (PC) and Radio Frequency (RF) front-ends. In our implementation, there is one PC hosting the eNB functions and another PC executing the EPC functions (i.e. Mobility Management Entity [MME], Serving / Packet Data Network Gateway [S/P-GW] and Home Subscriber Server 


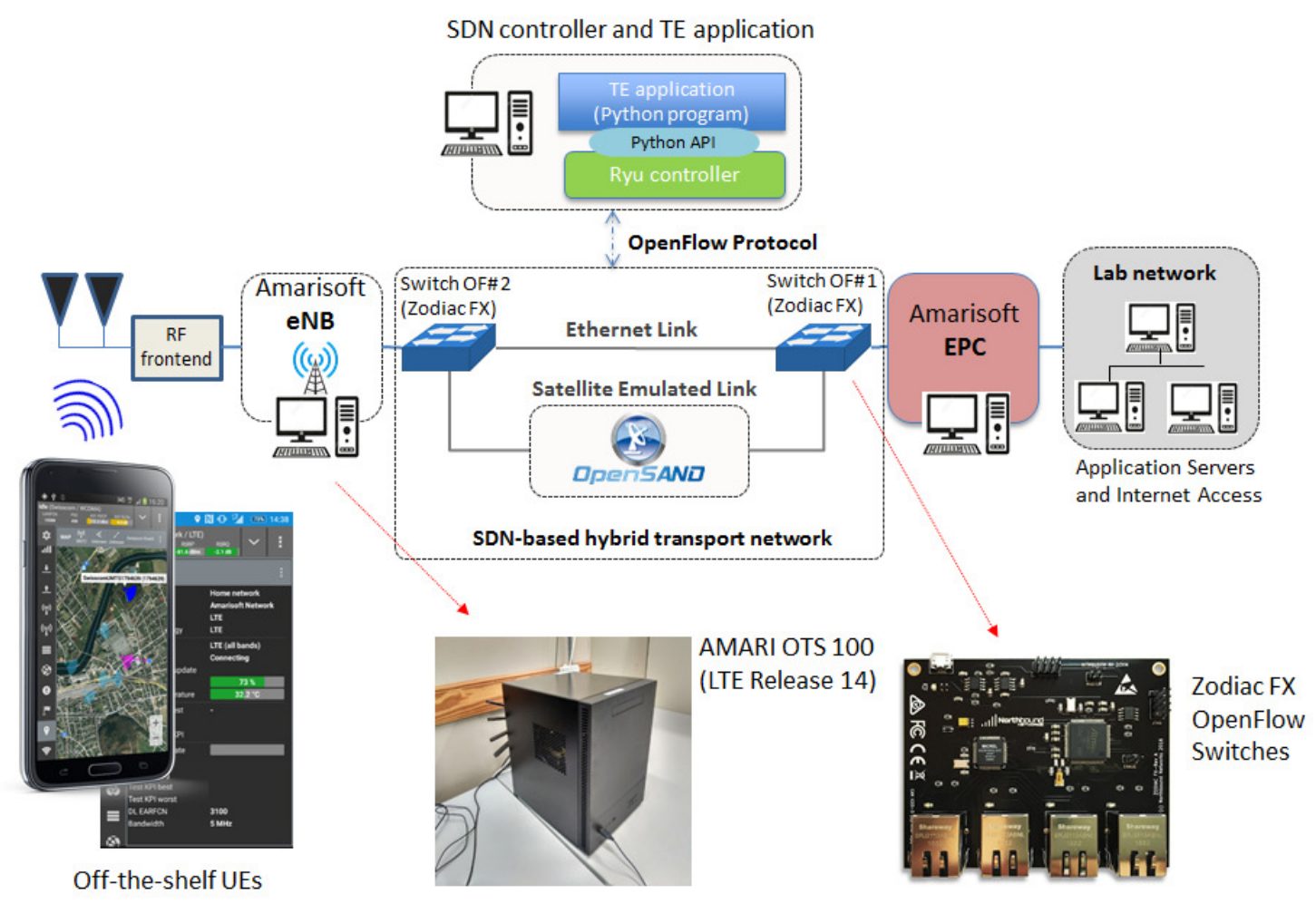

FIGURE 2 High level view of the experimental testbed components.

[HSS]). The LTE network is configured with a private network identity (i.e. a private Public Land Mobile Network [PLMN] identifier) and can be accessed by commercial LTE User Equipment (UE) such as smartphones and laptops fitted with USB data cards. Through the LTE network, UEs get IP connectivity to our laboratory network, where different application servers reside and from where access to the public Internet is also delivered.

With regard to the hybrid backhaul network between the eNB and EPC, it is built using switches OF, which stand for a particular realization of the generic NEs previously introduced in Fig. 1. There is one switch OF at each end with two possible connectivity paths between them. One of the two paths, referred to as the terrestrial path, is implemented with a direct wired Ethernet link between the two switches OF. The other path, referred to as the satellite path, goes through a satellite link emulator implemented with the OpenSAND system ${ }^{39}$. The way that both paths are exploited is controlled by a TE application programmed in a SDN controller based on the Ryu software package ${ }^{40}$. A more detailed description of the testbed components is addressed in the following, including the configuration settings used to carry out the operational validation and performance assessment reported in Section IV.

\section{1 | LTE Network and Terminals}

The private LTE network is built using the AMARI OTS 100 system ${ }^{38}$ deployed in two separate PCs. A detailed view of the hardware elements and interconnection arrangement of the testbed components is illustrated in Fig. 3 The two PCs running the AMARI OTS 100 system are tagged as eNB and EPC. The eNB PC is equipped with one PCIe RF board with four external antennas. The implementation is compliant with LTE Release 14, supporting the multiple frequency bands standardized below $6 \mathrm{GHz}$ and cell bandwidths of up to $20 \mathrm{MHz}$. Several SIM test cards have been provisioned into the Home Subscriber Server (HSS) database of the EPC PC so that UEs can gain connectivity to the laboratory network through the private LTE access. As illustrated in Fig. 3 the eNB and EPC PCs are attached to two separate subnets (192.168.1.X and 192.168.2.X) interconnected through the switches OF. On the other hand, the pool of IP addresses assigned to the UEs belongs to another subnet (192.168.20.X) from which the lab network is reached and internet access provided through Network Address Translation 


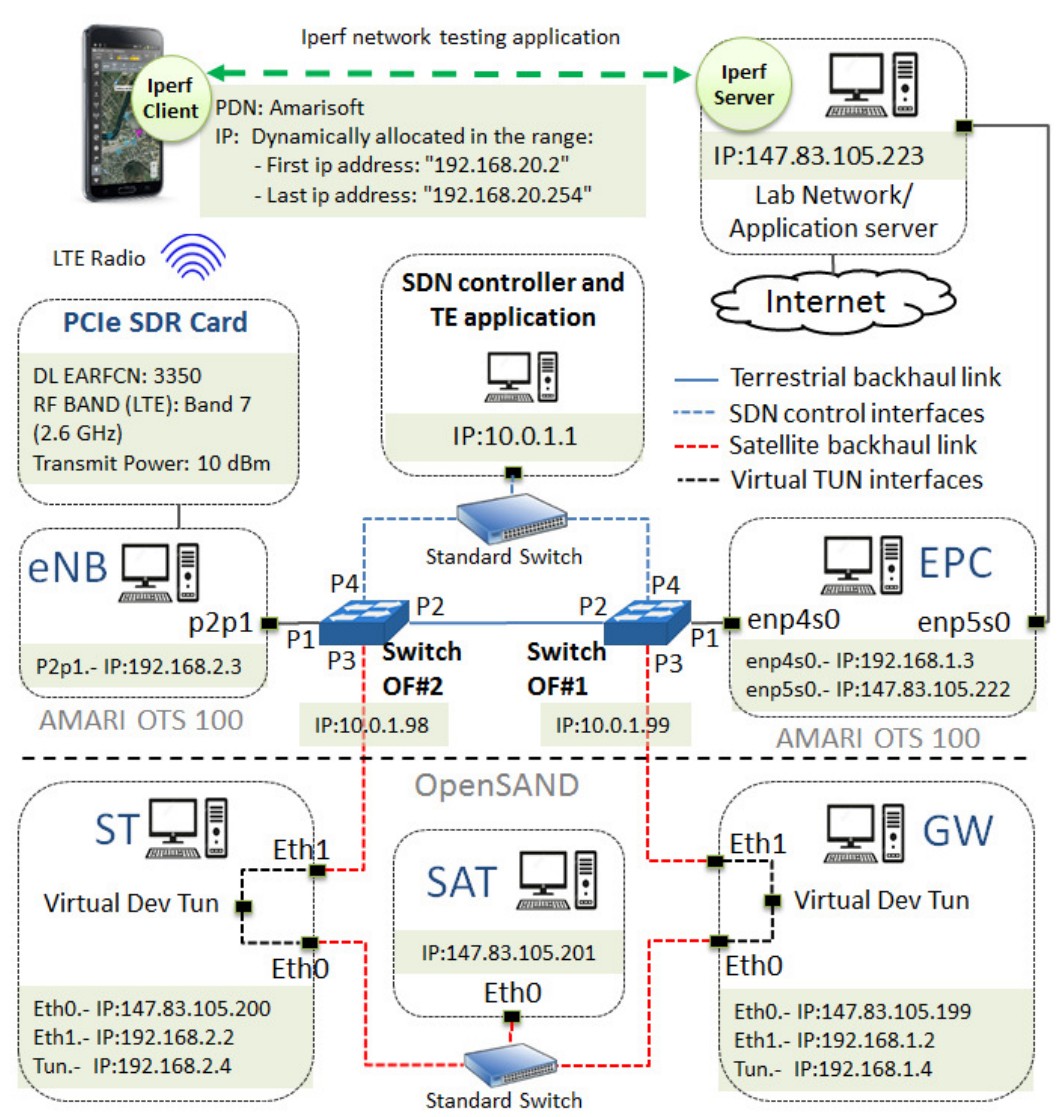

FIGURE 3 Detailed view of the hardware elements and interconnection settings among the testbed components.

(NAT) functionality in the EPC PC.

Table 1 provides the main configuration settings of this LTE network. Channel bandwidths of 5,10 and $20 \mathrm{MHz}$ are used in the experiments to enforce different ratios of satellite capacity over access capacity. UEs are placed at 1 meter distance from the eNB PC with line of sight to the PCI RF card. Regarding the Evolved Packet System (EPS) bearer service configuration that determines the QoS profile of IP connectivity service provided by the LTE network, the default configuration uses QoS Class Identifier (QCI) equal to 9, which corresponds to a non-guaranteed bit rate (N-GBR) service typically used in the commercial networks.

\section{2 | SDN-based hybrid backhaul network}

The data plane of the SDN-based hybrid backhaul network includes two Zodiac FX switches commercialized by NorthBound ${ }^{41}$. These are experimental-oriented switches OF equipped with 4 Fast Ethernet ports, one exclusively serving as a control interface port and the rest used for traffic forwarding. In particular, as illustrated in Fig. 3. port 4 (P4) of both switches OF is used for interaction with the SDN controller while the input/output traffic of the backhaul network is supported over P1. Using the OF protocol, the SDN controller can add, update, and delete flow entries in flow tables, both reactively (in response to new packets arriving at switches) and proactively. Each flow table in a switch OF contains a set of flow entries; each flow entry consists of match fields, counters, and a set of instructions and actions (e.g. packet forwarding actions, packet header modification actions) to apply to matching packets. Matching rules can be configured based on information such as ingress port and a combination of the multiple fields in packet headers (e.g. source/destination addresses, transport protocol, etc.). Zodiac FX switches support OpenFlow 1.3. 
TABLE 1 LTE Network Configuration

\begin{tabular}{l|l|l}
\hline & Feature / Parameter & Description and Values \\
\hline \multirow{5}{*}{ Network } & Supported 3GPP standard release & Release 14 \\
& Duplexing Mode & FDD \\
& Frecuency Band & LTE Band 7 $(2.6 \mathrm{GHz})$ \\
& Frecuency Channel & DL EARFCN \\
& Transmit Power & $<10 \mathrm{dBm}$ \\
& Channel BW & $5,10,20(\mathrm{MHz})$ \\
& Transmission Mode (TM) & TM1 \\
& Default EPS Bearer QCI & $9($ Non-GBR) \\
& Default EPS Bearer ARP & $15($ Lower Priority Level) \\
\hline \multirow{2}{*}{ Terminals } & UE & Samsung Galaxy S5 \\
& SIM Card & symoUSIM-SJS1 \\
\hline
\end{tabular}

The emulation of a satellite link is implemented with the OpenSAND platform, which is an open-source software tool that emulates a satellite communication system based on the DVB-S2/RCS standards ${ }^{39}$. Our implementation of OpenSAND uses three PCs, each hosting one of three different OpenSAND modules: a satellite emulator (SAT), a gateway (GW) and a satellite terminal (ST). As depicted in Fig. 3 the GW and the ST PCs are directly connected, respectively, to switches OF\#1 and OF\#2 for backhaul traffic forwarding through the emulated satellite link. Within the GW and ST, the backhaul traffic is captured/injected from/to the Ethernet network cards through TUN virtual network kernel interfaces so that the be re-directed through the SAT PC through a dedicated Ethernet switch for satellite emulation processing.

Table 2 provides the configuration settings of the hybrid backhaul network, detailing the considered OpenSAND configuration for the emulation of the forward and return link transfer rates and one-way latencies for different tested satellite types (e.g. GEO, MEO). The capacity of the satellite link has been defined according to the values of typical capacities offered commercially ${ }^{42}$. Likewise, the values of the latencies have been obtained from the parameters offered by current commercial systems, GEO and MEO (O3b system) shown in ${ }^{43}$.

TABLE 2 SDN-based hybrid backhaul network configuration

\begin{tabular}{l|l|l}
\hline & Feature / Parameter & Values \\
\hline Zodiac FX Switches & Operation Mode & OpenFlow 1.3 \\
\hline \multirow{5}{*}{ OpenSAND } & System type & DVB-S2/RCS \\
& Forward Link Symbol Rate & $20 \mathrm{E6}, 28.8$ E6 bauds \\
& Forward Link Modulation & QPSK \\
& Forward Link Coding Rate & $1 / 4,2 / 3$ \\
& Forward Link Capacity & $10,30 \mathrm{Mbps}$ \\
& Return Link CRA & $10 \mathrm{Mbps}$ \\
& Return Link Max RBDC & $1024 \mathrm{Kbps}$ \\
& Return Link Max VBDC & $55 \mathrm{Kbps}$ \\
& Return Link Capacity & $\sim 10 \mathrm{Mbps}$ \\
\cline { 2 - 3 } & One-way latency & $250 \mathrm{~ms}$ (for GEO at 36,000 Kms) \\
& & $70 \mathrm{~ms}$ (for MEO at $8,000 \mathrm{Kms})$ \\
\hline
\end{tabular}

${ }^{a}$ Constant Rate Allocation.

${ }^{b}$ Rate Based Dynamic Capacity. Assigned dynamically at the request of the Return Channel Satellite Terminal (RCST) according to the standard DVB-RCS.

${ }^{c}$ Volume Based Dynamic Capacity. Assigned dynamically at the request of the Return Channel Satellite Terminal (RCST) according to the standard DVB-RCS. 


\section{3 | SDN-Based TE Application}

The operation of the switches OF is commanded by a Ryu SDN controller, which is a component-based software fully written in Python. The Ryu controller exposes Application Programming Interfaces (API) for deploying network management and control applications as Python scripts ${ }^{40}$. Such APIs are a collection programming libraries that give access to the previously mentioned set of mechanisms supported by OpenFlow 1.3 so that capabilities for network state monitoring (e.g. switch status, port status, traffic load) and activation/ deactivation/ modification of flow tables in the switches OF are visible at application level.

The exposed API capabilities, allows us to program and demonstrate the operation of an SDN-based TE application able to deliver the sort of TE strategies studied in ${ }^{28}$. More specifically, the implemented SDN-based TE application is able to (1) learn the network topology, (2) monitor the network/port status, (3) detect new traffic at input/output ports of the backhaul network, (4) decide on the forwarding path based on a set of user-defined path computation policies and (5) enforce the desired forwarding path by populating the Flow Tables across the switches OF. An illustration of the implemented SDN-based TE application is given in Fig. 4 showing its internal organization and the exploited APIs. Of note is that the path computation policies are defined as a set of vectors where each row defines (1) Traffic matching conditions, including the traffic flow templates (e.g. discrimination of TCP, UDP, SCTP and/or ports of these protocols) and the input port of switches OF where the traffic flow is detected; (2) the network status of the switches OF ports (e.g. Up/Down); and (3) the preferred path policy (e.g. terrestrial or satellite path).

\section{4 | OPERATIONAL VALIDATION AND PERFORMANCE ASSESSMENT}

The operation of the testbed is validated through the execution of two illustrative TE procedures. The first one, referred to as path computation, shows how the implemented SDN-based TE application is able to enforce a desired routing over the hybrid backhaul each time a new LTE connection is to be established. On the other hand, the second procedure, called hereafter path restoration, shows how an unexpected failure of a link is handled by the SDN-based TE application so that the affected traffic flows are quickly re-directed over an alternative path. A description of the signaling flows and key decision-making points in the execution of the two procedures is firstly covered in this section. Next, two performance assessments are provided to complement the operational validation: one showing the impact on the LTE service performance of the routing decisions by the TE application and another addressing the impact of the link failure and path restoration procedure.

\subsection{Execution of path computation and restoration}

For the demonstration of the path computation procedure, the testbed is started with the configuration explained in the previous section and a LTE UE is switched on and registered in the LTE network. This results in the activation of the so called default EPS bearer service in the LTE network, which provides the basic IP connectivity service to the UE. Then, right after getting the connectivity service, an Iperf client application ${ }^{44}$ is launched in the UE so that UDP traffic begins to flow between the UE and an Iperf server located in the laboratory network. The routing of Iperf generated traffic through the hybrid backhaul is enforced by the TE application in the SDN controller based on the detection of the traffic type supported by the bearer service, the port where this traffic is detected and the network status. In particular, the path computation policy established for this scenario consists in forcing all traffic between the eNB and EPC (i.e. match condition: Traffic Type=Any, Input Port=P1 at both OF\#1 and $\mathrm{OF} \# 2$ ) to go through the terrestrial link (i.e. output port=P2 at both $\mathrm{OF} \# 1$ and $\mathrm{OF} \# 2$ ) whenever the backhaul network is operating under normal conditions (i.e. $\mathrm{P} 1 / \mathrm{P} 2 / \mathrm{P} 3$ status= $U p$ at both $\mathrm{OF} \# 1$ and $\mathrm{OF} \# 2$ switches). Otherwise, when one of the paths is not available (i.e. $\mathrm{P} 2$ or $\mathrm{P} 3=$ Down at $\mathrm{OF} \# 1$ or $\mathrm{OF} \# 2$ ), all the established flows are routed through the alternative path.

The results of the execution of the test are illustrated in Fig. 5 by showcasing the signaling exchanges between the testbed components with particular focus on the steps from the detection of the Iperf application traffic by the TE application at the SDN controller to the point at which the necessary flows are established in the switches OF for proper traffic forwarding through the backhaul links. All this information is collected with the help of the management tools of the AMARI OTS 100 and Zodiac FX switches and by inspecting traffic on the SDN controller, eNB and EPC with a network protocol analyzer tool (Wireshark).

The first step shown in Fig. 5 corresponds to the initial OF signaling handshaking between the SDN controller and the switches OF for the activation of the control plane. This signaling goes through the P4 ports and consequently does not traverse 

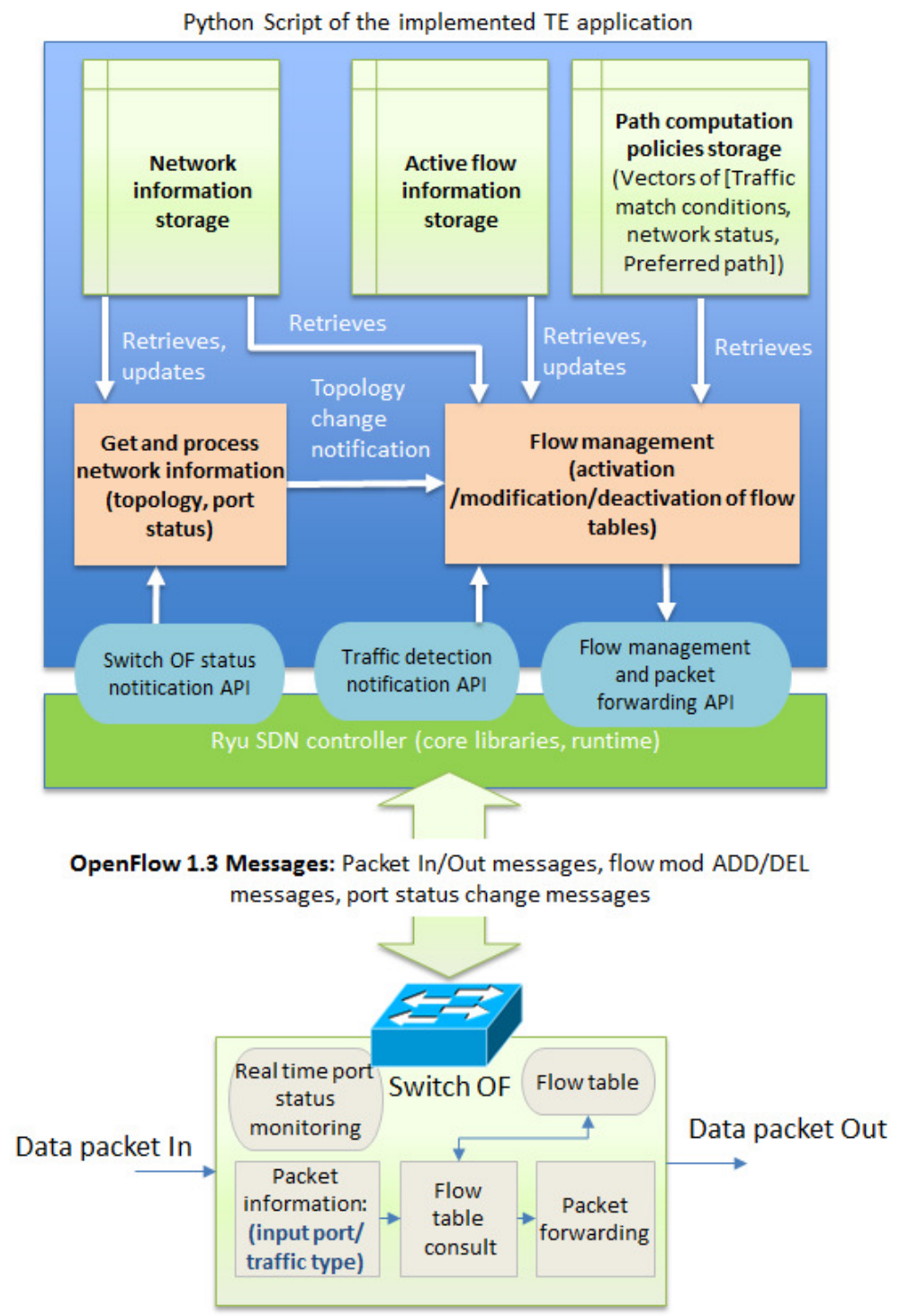

FIGURE 4 Illustration of the implemented SDN-based TE application.

the hybrid backhaul data plane. The second step corresponds to the initial signaling exchange between the eNB and the EPC. This signaling, commonly denoted as S1 Setup and which uses S1 Application Protocol (S1AP) and Stream Control Transmission Protocol (SCTP) as transport protocol, is necessary for the activation of the control plane of the S1 interface between the eNB and the EPC components of the LTE network. Unlike the previous mentioned OF signaling, now the S1AP signaling is indeed transferred over the hybrid backhaul network, so that the TE application detects this traffic in both directions and creates the corresponding flows (i.e. flows 1,3,5 and 7 in Table 3). Next, once the $\mathrm{S} 1$ interface is operational, the step 3 in Fig. 5 corresponds to the network registration and activation of the default EPS bearer service when the UE is switched on. In this case, the signaling originated in step 3 between the eNB and EPC is sent through the path previously established in the activation of the control plane of S1, so that no new flows are created at this point. Reached this situation, the Iperf traffic is started at step 4. This result in a new IP packet detected at OF\#2 for which no matching flow entry exists (step 5 and 6). The detected packet is indeed a GPRS Tunneling Protocol for User Plane (GTP-U) packet that encapsulates the original UDP packet received from the UE. As a result, the TE application is notified in step 7 of the presence of new traffic. This triggers the computation of the routing path at step 8, where, according to the established path routing policy, the creation of a new flow (flow 6 in Table 3 ) is decided. This decision triggers the activation of the flow through the OF protocol signaling (step 9 and 10) together with a specific OF command (step 11) given to the switch to forward the packet that triggered the flow creation. Once the forwarded 


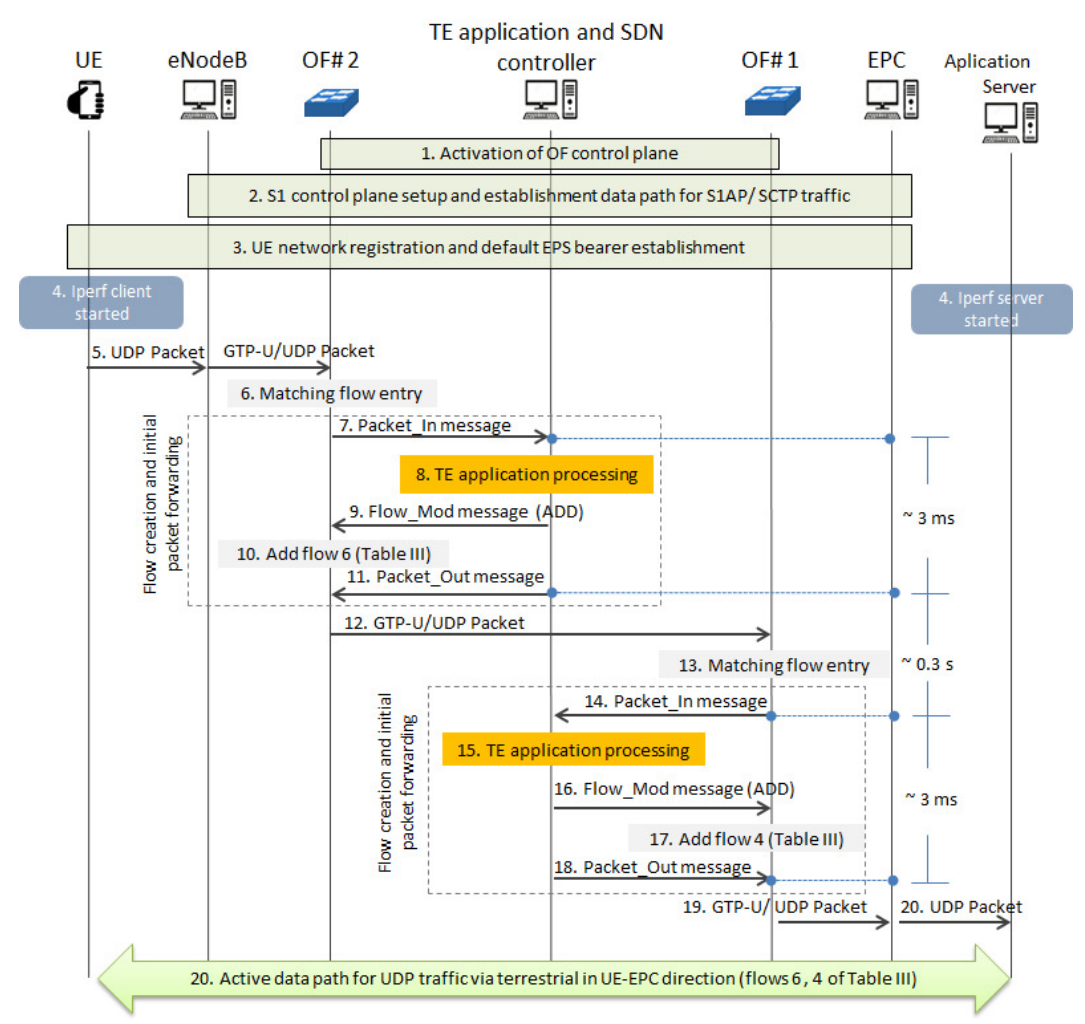

FIGURE 5 Signalling exchange for the path computation procedure.

GTP-U/UDP packet reaches OF\#1, the same process of creation of a flow in OF\#2 is now repeated for OF\#1 (steps 14 to 18), leading to the creation of a new flow (flow 4 in Table 3 ) and to the eventual forwarding of the GTP-U/UDP packet towards the EPC (step 19). Finally, the original UDP packet is decapsulated in the EPC and, after NAT processing, forwarded to the Iperf server within the laboratory network. At this point, the connectivity path in one direction is fully operational between the eNB and EPC so that subsequent packets will already match the created flow entries and therefore be forwarded without requiring any interaction with the SDN controller. Later, when traffic is detected in the opposite direction, the process is repeated concluding with the creation of the flows 2 and 8 in Table 3 Fig. 5 also shows that the overall processing time for flow creation including the decision-making process of the TE application is roughly $\sim 3 \mathrm{~ms}$ measured from the arrival of the new traffic notification (step 7) to the sending of the output packet command (step 11). On the other hand, the response time between the eventual traffic forwarding at OF\#2 until the notification of the detection of the packet at OF\#1 (step 14) results in the order of $\sim 300 \mathrm{~ms}$. Of note is that this response time is determined by the switch manufacturer design, which is to be much lower in a carrier-grade product. It is also worth noting that the demonstrated TE application, while being illustrative of the benefits achievable with a SDN-enabled framework, may admit different optimizations and trade-offs between the achieved network performance and the complexity of the TE application logic. For instance, in the presented procedure, the creation of flow 4 in OF\#1 could have been triggered at the same time that flow 6 was created, thus saving the flow creation time of $3 \mathrm{~ms}$ in OF\#1. This would be a more proactive approach to handle the flows, creating them before traffic is actually detected in the switches, in contrast to the more reactive approach followed in the implemented TE application logic. However, the implementation of this proactive approach would come at the expenses of a bit more sophisticated TE application logic, which should ensure full consistency of flows creation / deletion across the network. The flows created for traffic S1-AP/SCTP and GTP-U during the whole Path Computation process are summarized in Table 3 , totaling 8 different flows (4 at each switch OF).

For illustrative purposes, Fig. 6 shows a Wireshark capture at the PC running the SDN controller with the signalling messages originated between Steps 7, 9, 11, 14, 16 and 18 of message chart of Fig. 5 With the same purpose, Fig. 7 shows the information provided by the Zodiac FX management tool regarding to the configuration of one of these flows. 
TABLE 3 Flows created during the path computation and restoration procedures

\begin{tabular}{|c|c|c|c|c|}
\hline Procedure & Switch & Flow & In Port/Out Port & Traffic Type \\
\hline \multirow{8}{*}{ Path Computation } & \multirow{4}{*}{ OF\#1 } & 1 & $\mathrm{P} 1 / \mathrm{P} 2$ & S1-AP/SCTP \\
\hline & & 2 & $\mathrm{P} 1 / \mathrm{P} 2$ & GTP-U/UDP \\
\hline & & 3 & $\mathrm{P} 2 / \mathrm{P} 1$ & S1-AP/SCTP \\
\hline & & 4 & $\mathrm{P} 2 / \mathrm{P} 1$ & GTP-U/UDP \\
\hline & \multirow{4}{*}{$\mathrm{OF} \# 2$} & 5 & $\mathrm{P} 1 / \mathrm{P} 2$ & S1-AP/SCTP \\
\hline & & 6 & $\mathrm{P} 1 / \mathrm{P} 2$ & GTP-U/UDP \\
\hline & & 7 & $\mathrm{P} 2 / \mathrm{P} 1$ & S1-AP/SCTP \\
\hline & & 8 & $\mathrm{P} 2 / \mathrm{P} 1$ & GTP-U/UDP \\
\hline \multirow{8}{*}{ Path Restoration } & \multirow{4}{*}{ OF\#1 } & 9 & $\mathrm{P} 1 / \mathrm{P} 3$ & S1-AP/SCTP \\
\hline & & 10 & $\mathrm{P} 1 / \mathrm{P} 3$ & GTP-U/UDP \\
\hline & & 11 & $\mathrm{P} 3 / \mathrm{P} 1$ & S1-AP/SCTP \\
\hline & & 12 & $\mathrm{P} 3 / \mathrm{P} 1$ & GTP-U/UDP \\
\hline & \multirow{4}{*}{$\mathrm{OF} \# 2$} & 13 & $\mathrm{P} 1 / \mathrm{P} 3$ & S1-AP/SCTP \\
\hline & & 14 & $\mathrm{P} 1 / \mathrm{P} 3$ & GTP-U/UDP \\
\hline & & 15 & $\mathrm{P} 3 / \mathrm{P} 1$ & S1-AP/SCTP \\
\hline & & 16 & $\mathrm{P} 3 / \mathrm{P} 1$ & GTP-U/UDP \\
\hline
\end{tabular}

\begin{tabular}{|c|c|c|c|c|}
\hline Time & Source & Destination & Protocol & Length Info \\
\hline 17.6739292 & 10.0 .1 .98 & 10.0 .1 .1 & OpenFlow & 214 Type: OFPT_PACKET_IN \\
\hline 17.6765547 & 10.0 .1 .1 & 10.0 .1 .98 & OpenFlow & 174 Type: OFPT_FLOW_MOD \\
\hline 769505 & 10.0 .1 .1 & .0 .1 .98 & low & 228 Type: OFPT_PACKET_OUT \\
\hline 36365 & 10.0 .1 .99 & 10.0 .1 .1 & OpenFlow & 206 Type: OFPT_PACKET_IN \\
\hline 63060 & 10.0 .1 .1 & 10.0 .1 .99 & OpenFlow & 174 Type: OFPT_FLOW_MOD \\
\hline 17.9666130 & 10.0 .1 .1 & 10.0 .1 .99 & OpenFlow & 220 Type: OFPT_PACKET_OUT \\
\hline
\end{tabular}

FIGURE 6 OF messages originated in the steps 7, 9, 11 and 14, 16, 18 illustrated in Fig. 5 .

For the demonstration of the path restoration procedure, the testbed is started as in the previous demonstration and, once the connectivity is operational and the Iperf traffic is flowing, a link disconnection is manually forced. Fig. 8 shows the signalling originated as a result of this disconnection when the affected path is the terrestrial one.

As seen in Fig. 8, the link failure event is detected at OF\#2 that changes the status of P2 (from $U p$ to Down) and sends an OF message (Step 2) to the SDN controller (it should be noted that in this case, the link event is detected at both switches OF, so the port status change notification actually arrive from both switches OF, even though Fig. 8 only shows the link failure event detected only at OF\#2). This triggers a notification to the TE application that checks which flows at both switches OF are affected by the disconnection (i.e. all flows that have as output port $=\mathrm{P} 2$ ). Accordingly, the TE application triggers the deletion of these flows between steps 4 and 11 (i.e. flows 1, 2, 5 and 6 in Table 3 ) so that traffic forwarding towards the impaired path is stopped. In terms of execution times, Fig. 8 shows a response time in the order of 1 second between the link disconnection and the reception of the port status message at the SDN controller, which is again attributable to the specific hardware used in the testbed. On the other hand, the process involving the TE application decision-making and the deletion of the flows remains in the order of a few milliseconds.

After the removal of the flows, the establishment of the new path is solved in the implemented TE application by reactively triggering the creation of new flows when notifications about packets not finding a flow matching entry are received in the SDN controller. This process is indeed the same as the one followed in the path computation procedure previously explained in Fig. 5 The main difference is that now network conditions are different (i.e. P2 is down) so that the TE application decides creating the new flows using the satellite path. The new flows created in this case are listed as flows 9 to 16 in Table 3 


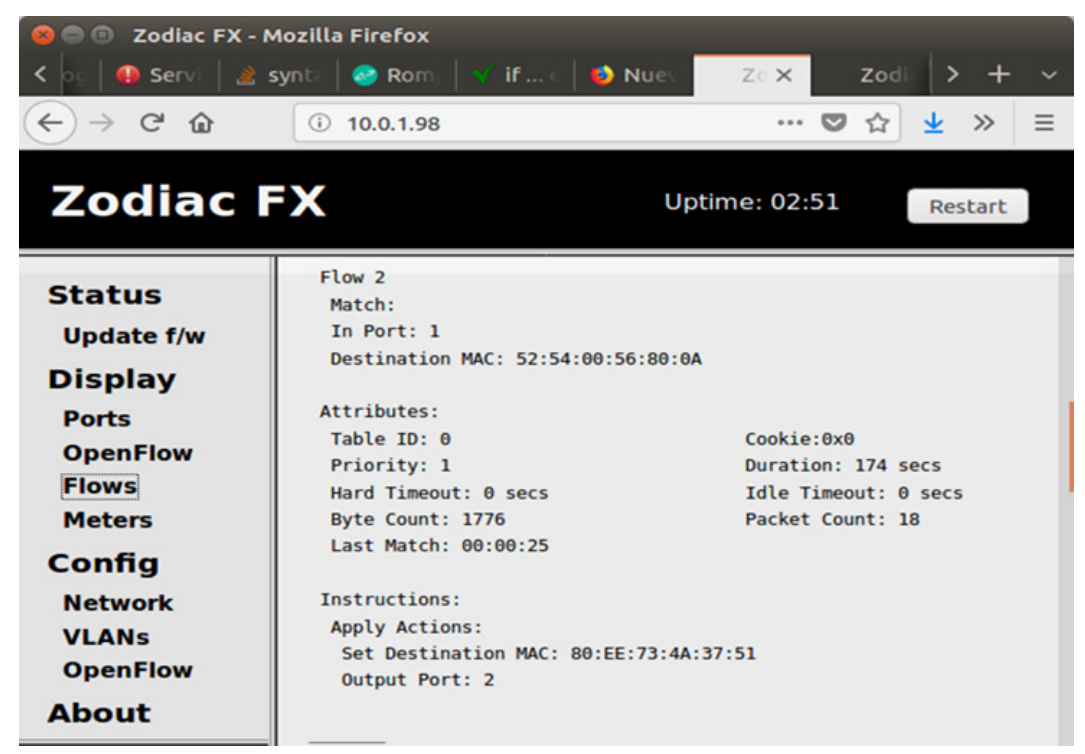

FIGURE 7 Illustration of the flow attributes information as displayed by the Zodiac FX switches management tool.

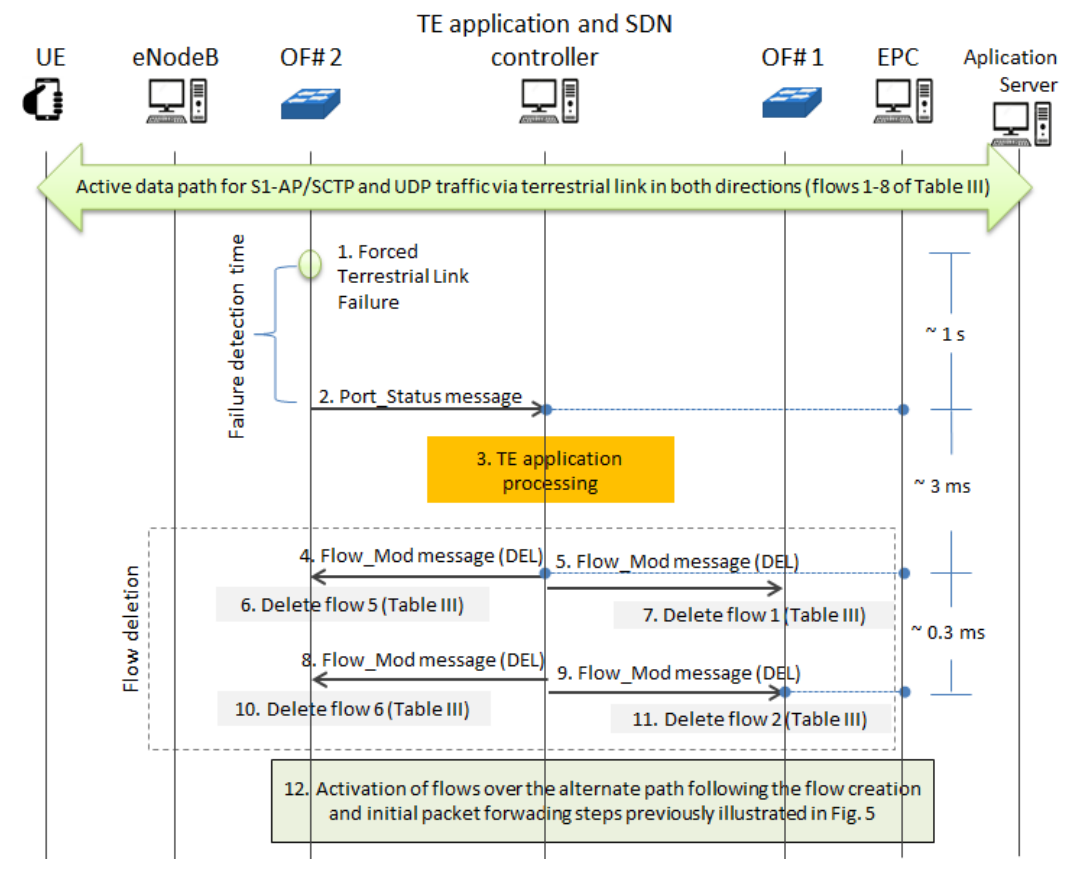

FIGURE 8 Signalling exchange for the path restoration procedure.

For illustrative purposes, Fig. 9 shows a Wireshark capture of the message sequence from the reception of port status change notification message from OF\#2 at the SDN controller, followed by four OFPT_FLOW_MOD messages sent by the SDN controller to the switches OF\#1 and OF\#2 in order to delete the previously referred flows. It's worth noting that the OF protocols use the same type of OFPT_FLOW_MOD messages for both creation and removal of flows, though internal headers define whether it is an OFPFC_ADD or OFPFC_DELETE operation, as illustrated in Fig. 10 
it could be seen that UDP throughput limitation is basically a matter of capacity limitation. Therefore, long-lived UDP connections are clear candidates to be offloaded through the satellite capacity.

The situation gets more complex when it comes to TCP traffic. In general, TCP throughput is lower than the UDP throughput due to the overhead caused by the TCP congestion control mechanisms in order to cope with packet losses $\dot{H}^{-}$This throughput reduction is clearly more accentuated with higher latencies in the satellite links. In particular, when considering a satellite capacity of $10 \mathrm{Mbps}$, the average throughput rate remains around 7.06.5-7.69 Mbps (in contrast to the 8.6 Mbps achieved with UDP), which is expectedly limited by the satellite capacity. However, when considering a higher satellite link capacity (30 Mbps), the throughput achieved through the LTE network does not improve under the MEO configuration, yielding around $7 \mathrm{Mbps}$ irrespective of the LTE channel bandwidth (in contrast with the 17.4-27.2 Mbps of UDP), and even gets worse for the GEO case, yielding around 5.5 Mbps also regardless of channel bandwidth. A more detailed analysis of this behavior, out of the scope of this paper, has revealed that the low TCP throughputs are actually due to a high fluctuation observed in the instantaneous TCP throughput, with sharp drops that bring down the averaged values.

TABLE 4 LTE service performance under different satellite path configurations.

\begin{tabular}{|c|c|c|c|c|c|c|c|}
\hline & & & \multirow{3}{*}{$\begin{array}{c}\text { Benchmark } \\
\text { Configuration } \\
\text { (100 Mbps Terretrial } \\
\text { Capacity) }\end{array}$} & \multicolumn{4}{|c|}{ Satellite Path Configurations } \\
\hline & & & & \multicolumn{2}{|c|}{$\begin{array}{c}\text { Forward Link Capacity } \\
=10 \mathrm{Mbps}\end{array}$} & \multicolumn{2}{|c|}{$\begin{array}{c}\text { Forward link Capacity } \\
=30 \mathrm{Mbps}\end{array}$} \\
\hline & & & & MEO & GEO & MEO & GEO \\
\hline \multirow{3}{*}{$\begin{array}{c}\text { LTE } \\
\text { Channel } \\
\text { Bandwidth }\end{array}$} & $5 \mathrm{MHz}$ & $\begin{array}{l}\text { RTT } \\
\text { TCP } \\
\text { UDP }\end{array}$ & $\begin{array}{c}30.63 \mathrm{~ms} \\
16.5 \mathrm{Mbps} \\
17.5 \mathrm{Mbps}\end{array}$ & $\begin{array}{c}232.5 \mathrm{~ms} \\
7.37 \mathrm{Mbps} \\
8.6 \mathrm{Mbps}\end{array}$ & $\begin{array}{c}591.1 \mathrm{~ms} \\
7.06 \mathrm{Mbps} \\
8.6 \mathrm{Mbps}\end{array}$ & $\begin{array}{c}229.1 \mathrm{~ms} \\
7.1 \mathrm{Mbps} \\
17.4 \mathrm{Mbps}\end{array}$ & $\begin{array}{c}579.7 \mathrm{~ms} \\
\text { 5.67 Mbps } \\
17.4 \mathrm{Mbps}\end{array}$ \\
\hline & $10 \mathrm{MHz}$ & $\begin{array}{l}\text { RTT } \\
\text { TCP } \\
\text { UDP }\end{array}$ & $\begin{array}{c}\text { 36.12 ms } \\
\text { 34.7 Mbps } \\
\text { 35.9 Mbps }\end{array}$ & $\begin{array}{c}231.6 \mathrm{~ms} \\
7.56 \mathrm{Mbps} \\
8.6 \mathrm{Mbps}\end{array}$ & $\begin{array}{c}581.9 \mathrm{~ms} \\
7.38 \mathrm{Mbps} \\
8.6 \mathrm{Mbps}\end{array}$ & $\begin{array}{l}236.7 \mathrm{~ms} \\
6.46 \mathrm{Mbps} \\
27.2 \mathrm{Mbps}\end{array}$ & $\begin{array}{c}577.8 \mathrm{~ms} \\
5.53 \mathrm{Mbps} \\
27.2 \mathrm{Mbps}\end{array}$ \\
\hline & $20 \mathrm{MHz}$ & $\begin{array}{l}\text { RTT } \\
\text { TCP } \\
\text { UDP }\end{array}$ & $\begin{array}{l}35.11 \mathrm{~ms} \\
71.9 \mathrm{Mbps} \\
59 \mathrm{Mbps}\end{array}$ & $\begin{array}{c}239.8 \mathrm{~ms} \\
7.69 \mathrm{Mbps} \\
8.6 \mathrm{Mbps}\end{array}$ & $\begin{array}{c}590.1 \mathrm{~ms} \\
7.51 \mathrm{Mbps} \\
8.6 \mathrm{Mbps}\end{array}$ & $\begin{array}{c}234.7 \mathrm{~ms} \\
\text { 7.17 Mbps } \\
27.2 \mathrm{Mbps}\end{array}$ & $\begin{array}{c}580.1 \mathrm{~ms} \\
5.4 \mathrm{Mbps} \\
27.2 \mathrm{Mbps}\end{array}$ \\
\hline
\end{tabular}

\subsection{Impact of the path restoration procedure on the LTE service}

Finally, the impact on the LTE service performance of a backhaul link failure and the subsequent execution of the path restoration procedure is analyzed in this section. To that end, the LTE channel bandwidth is set to $10 \mathrm{MHz}$ and OpenSAND is configured with a forward link capacity of $30 \mathrm{MHz}$ and GEO-type latency. When the testbed is started, the Iperf application is used to transfer UDP traffic at $15 \mathrm{Mbps}$. Such traffic is initially routed through the terrestrial path and, at a given point in time, a terrestrial link failure is provoked and the TE application takes care of re-routing the UDP traffic through the satellite path following the path restoration procedure previously analyzed. Then, after a few seconds of sending the traffic over the satellite link, the terrestrial link is turned up again and the TE application triggers again a path restoration procedure now to move back the UDP traffic over the terrestrial path. An illustration of the tested behavior is shown in Fig. 11 as measured and visualized from the Amarisoft management tool at the LTE eNB. The assessment has been carried out under different UDP transfer rate configurations (i.e. 5, 15 and $25 \mathrm{Mbps}$ ). Each configuration has been executed 10 times for statistics gathering.

\footnotetext{
${ }^{\dagger}$ Except for the configuration with an LTE channel bandwidth of $20 \mathrm{MHz}$. The reason has only to do with the way that we are computing the maximum UDP throughout. In particular, given that the maximum UDP rate is defined as the highest bit rate that can be configured with a packet loss ratio not exceeding $1 \%$, the experiments show that in this case the $1 \%$ ratio is reached with relatively low UDP bit rates rate (59 Mbps) and increases smoothly from this point with higher UDP bit rates. On the other hand, it is observed that TCP connections can still properly cope with this range of packet losses given the low latency and high capacity of the backhaul link used in this configuration.
} 


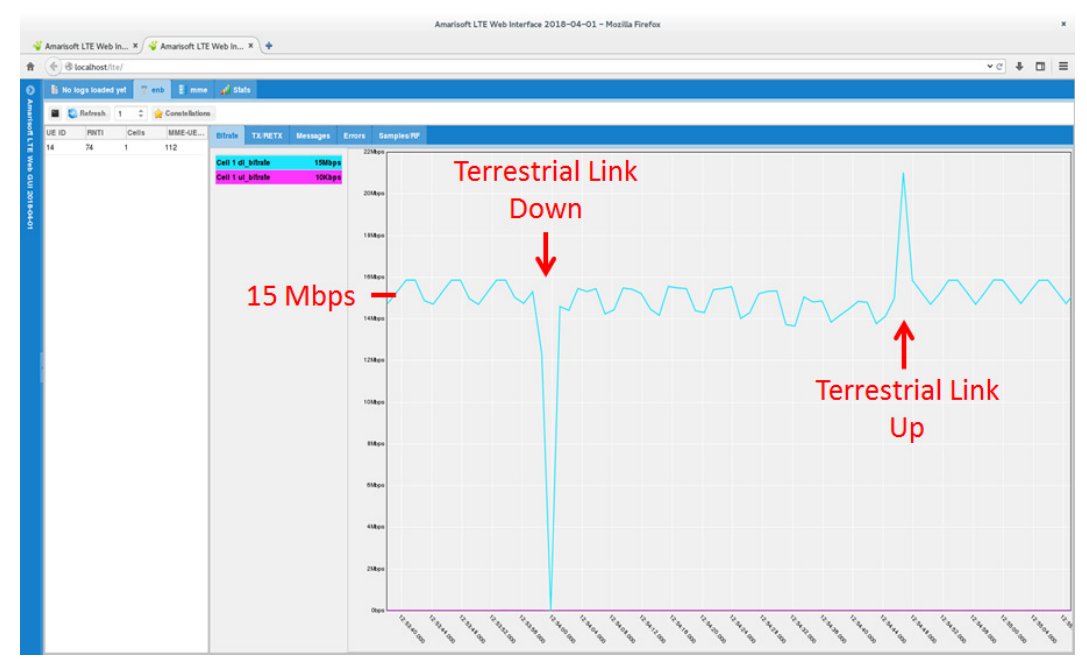

FIGURE 11 Time evolution of the UDP throughput during the test, highlighting the points at which terrestrial link goes down and up again.

Focusing first on the link failure event, it could be seen in Fig. 11 that the time taken for the path restoration procedure causes a noticeable throughput drop. A quantification of this traffic drop is provided in Table 5 in terms of the number of datagrams lost for 5, 15 and $25 \mathrm{Mbps}$ UDP traffic rate. As it can be observed from the table, the amount of datagrams lost is directly proportional to the bit rates and the path restoration time, which is of the order of 1 second in our setting due to the response time of the utilized OF switching hardware. Therefore, the impact due to the processing time associated with the operation of the TE application is almost negligible. As a side note to further stress the advantage of the implemented TE application, turning the operation mode of OF\#1 and OF\#2 from "OpenFlow" to "standard", that is, letting the re-direction of traffic in the backhaul to be fixed by the conventional distributed spanning tree protocol supported in the switches, and repeating the same experiment, observed interruption times boost above tens of seconds.

Moving now to the point that the terrestrial path goes operational again and the traffic is switched back to this path by the TE application, no packet losses are observed in this case. This is because the traffic can be still correctly transferred through the satellite link which remains operational during the path switching process. Remarkably, this situation even results here in a temporary increase of the throughput due to the simultaneous arrival of traffic via both links. However, since the satellite link latency is higher, the first packets sent through the terrestrial link are out of order. A quantitative assessment of this effect is also presented in Table 5. where it can be seen that the amount of datagrams received out of order is proportional to the UDP application bit rate and the satellite latency.

TABLE 5 Impact of the path restoration procedure on LTE service performance.

\begin{tabular}{l|c|c|c}
\hline & $\begin{array}{c}\text { Iperf Bit Rate (Mbps)/ } \\
\text { Datagrams per sec }\end{array}$ & $\begin{array}{c}\text { Datagrams Lost } \\
\text { (Avg./ } \sigma)\end{array}$ & $\begin{array}{c}\text { Datagrams received } \\
\text { out of order (Avg./ } \sigma \text { ) }\end{array}$ \\
\hline \multirow{3}{*}{ Terrestrial Link from Up to Down } & $5 / 425$ & $465 / 211$ & - \\
& $15 / 1275$ & $1566 / 674$ & - \\
\hline \multirow{3}{*}{ Terrestrial Link from Down to Up } & $25 / 2125$ & $2150 / 615$ & - \\
\hline & $5 / 425$ & - & $99.7 / 5.69$ \\
& $15 / 1275$ & - & $204.2 / 55.8$ \\
\hline
\end{tabular}




\section{5 | CONCLUSIONS}

The adoption of SDN technologies is nowadays acknowledged as a key enabler for a better integration of a satellite component into 5G networks. Sustained in the architectural designs proposed by the EU-funded VITAL project, this paper has presented an experimental PoC and validation of an integration solution based on the use of SDN technologies for the realization of E2E TE applications in hybrid terrestrial-satellite backhaul mobile scenarios.

The implemented testbed has allowed us to assess the feasibility of the proposed SDN-based integration solution under a practical laboratory setting that combines the use of commercial, experimentation-oriented and emulation equipment and software. Procedures for path computation and restoration have been showcased, assessing the incurred execution times and signalling exchanges incurred in the different steps. In particular, it has been demonstrated that the time incurred in the creation and deletion of flows from the controller is of the order of a few milliseconds, and that the longest delays incurred in the tested procedures that actually have a noticeable impact on service performance are mainly associated with the nature of the hardware used in the experimentation. Moreover, it has been also validated the versatility of using a high-level language like Python and the existing OF libraries in the Ryu controller for programming the TE application in order to enforce different traffic routing and path failure restoration policies. Finally, the testbed has also allowed us to assess the impact on the LTE service performance for TCP and UDP traffic flows when using and switching to/from a satellite link.

While the results achieved in this PoC are clearly supportive of the potential and feasibility of applying SDN technologies for improved satellite-terrestrial integration, further work is still necessary to demonstrate improved TE application logic and the use of additional control features enabled by OF. In particular, features such as per-flow metering, rate-limiting, packet duplication for multicast, etc., could be leveraged for the implementation of more complex traffic distribution and QoS policing operations.

\section{ACKNOWLEDGMENT}

This work has been supported by the Spanish Research Council and FEDER funds under SONAR 5G grant (ref. TEC201782651-R)

\section{References}

1. 5G Vision. The 5G Infrastructure Public Private Partnership: the next generation of communication networks and services. https://5g-ppp.eu/wp-content/uploads/2015/02/5G-Vision-Brochure-v1.pdf. February 2015.

2. NGMN Alliance, 5G White Paper. https://5g-ppp.eu/wp-content/uploads/2015/02/5G-Vision-Brochure-v1.pdf. February 2015.

3. NetWorld2020's. SatCom WG The role of satellites in 5G. https://www.networld2020.eu/wpcontent/uploads/2014/02/SatCom-in-5G_v5.pdf. July 2014; Version 5

4. Sacchi, C.; Bhasin, K.; Kadowaki, N.; Vong, F. Toward the "space 2.0" Era [Guest Editorial]. IEEE Communications Magazine. March 2015 ; volume(53). no.3: pages 16-17.

5. Osseiran A. et al. Scenarios for $5 G$ Mobile and Wireless Communications: The Vision of the METIS Project. IEEE Communications Magazine. May 2014; pages 26-35.

6. Evans, B. et al. The role of satellites in 5G. 23rd European Signal Processing Conference (EUSIPCO). September 2015.

7. Corici, M. et al. Assessing satellite-terrestrial integration opportunities in the 5 G environment.. September 2016. Available online at https://artes.esa.int/sites/default/files/Whitepaper

8. Fenech, H.; Amos, S.; Tomatis, A.; Soumpholphakdy, V. High throughput satellite systems: An analytical approach. in Aerospace and Electronic Systems, IEEE Transactions. Jan 2015 ; volume(51). no.1: pages 192-202. 
9. ARTES programme. ESA announces dedicated support for the development of megaconstellations. https://artes.esa.int/news/esa-announces-dedicated-support-development-megaconstellations. Last updated at 02 February 2016.

10. 3GPP TS 22.261 V0.1.1 (2016-08). Service requirements for next generation new services and markets. Stage 1 (Release 15). August 2016.

11. 3GPP a Global Initiative, The Mobile Broadband Standard. Satellite components for the 5G system. http://www.3gpp.org/news-events/3gpp-news/1933-sat_ntn. Last updated at January 4, 2018

12. Watts S., Glenn O. $5 G$ resilient backhauling using integrated satellite networks. Advanced Satellite Multimedia System Conference. September 2014.

13. Gomes T. et al. A survey of strategies for communication networks to protect against large-scale natural disasters. 8th International Workshop on Resilient Networks Design and Modeling (RNDM). 13-15 Sept. 2016; Halmstad, Sweden.

14. Mendoza F., Ferrús R., Sallent O. Flexible Capacity and Traffic Management for Hybrid Satellite-Terrestrial Mobile Backhauling Networks. International Symposium on Wireless Communication Systems (ISWCS). September 2016; Poznan, Poland.

15. Mendoza F., Ferrús R., Sallent O. A traffic distribution scheme for $5 G$ resilient backhauling using integrated satellite networks. 13th International Wireless Communications and Mobile Computing Conference (IWCMC). June 2017; Valencia, Spain.

16. Breiling M. et al. LTE Backhauling Over MEO-Satellite. Advanced Satellite Multimedia Systems Conference. IEEE. September 2014; pages 174-181.

17. Bertaux L. et al. Software Defined Networking and Virtualization for Broadband Satellite Networks. IEEE Communications Magazine. March 2015; pages 54-60.

18. Ferrús R., Koumaras H., Sallent O., Agapiou G., Rasheed T., Kourtis M.-A., Boustie C., Gelard P., Ahmed T. SDN/NFVenabled satellite communications networks: Opportunities, scenarios and challenges. Elsevier, Physical Communication. March 2016; volume(18). Part 2: pages 95-112.

19. H2020 VITAL Project. VIrtualized Hybrid Satellite-Terrestrial systems for resilient and flexible future networks. Project website at http://www.ict-vital.eu/. Start date 1 February 2015.

20. Toufik A. et al. Software-defined satellite cloud RAN. International Journal of Satellite Communications and Networking. February 2017; volume(36). Issue 1: pages 108-133.

21. H2020 SaT5G Project. Satellite and Terrestrial Network for 5G, Start date June 2017. Project website at http://sat5gproject.eu/ . Start date June 2017.

22. ARTES Telecommunications \& Integrated Applications. Satellite for 5G. Available onlinte at http://www.esa.int/Our_Activities/Telecommunications_Integrated_Applications/Satellite_for_5G. Last update at 21 June 2017.

23. Giambene G., Kota S. and Pillai P. Satellite-5G Integration: A Network Perspective. in IEEE Network. September/October 2018; volume(32). no.5: pages 25-31.

24. Xu S., Wang X. and Huang M. Software-Defined Next-Generation Satellite Networks: Architecture, Challenges, and Solutions. in IEEE Access. 2018; volume(6): pages 4027-4041.

25. Li T., Zhou H., Luo H. and Yu S. SERvICE: A Software Defined Framework for Integrated Space-Terrestrial Satellite Communication. in IEEE Transactions on Mobile Computing. 1 March 2018; volume(17). no.3: pages 703-716.

26. Ferrús R., Sallent O., Ahmed T., Fedrizzi R. Towards SDN/NFV-enabled satellite ground segment systems: End-to-End Traffic Engineering Use Case. IEEE International Conference on Communications Workshops (ICC Workshops). 21-25 May 2017; Paris. 
27. Mendoza F., Ferrús R., Sallent O. SDN-enabled SatCom Networks for Satellite-Terrestrial Integration. volume of Satellite Communications in the 5G Era. Chapter 3: pages 61-99; address: IET Digital Library. edition ed. 2018. ISBN: 978-178561-427-9.

28. Mendoza F., Ferrús R., Sallent O. SDN-based Traffic Engineering for Improved Resilience in Integrated SatelliteTerrestrial Backhaul Networks. 4th International Conference on Information and Communication Technologies for Disaster Management (ICT-DM). 21-25 Dec. 2017; MÃijnster, Germany.

29. 3GPP TS 23.214. Architecture enhancements for control and user plane separation of EPC nodes. Release 14 . June 2016.

30. 3GPP TS 23.501. System Architecture for the 5G System. Release 15, December 2016.

31. ETSI TR 101.984. âĂIJSatellite Earth Stations and Systems (SES); Broadband Satellite Multimedia (BSM); Services and architecturesâ̆̆İ. December 2007.

32. Abdellatif et al. Exposing an Openflow Switch Abstraction of the Satellite Segment to Virtual Network Operators. IEEE 83rd Vehicular Technology Conference (VTC Spring). 2016; Nanjing.

33. Open Networking Foundation. Functional Requirements for Transport API. ONF TR-527. 10 June 2016.

34. Open Networking Foundation. Microwave Information Model. ONF TR-532. Version 1.0, December 2016.

35. Zhang X. et al. Applicability of YANG models for Abstraction and Control of Traffic Engineered Networks. draft-zhangteas-actn-yang, work in progress.

36. ONF Open Networking Foundation. OpenFlow Switch Specification. Version 1.3.0 (Wire Protocol 0x04). 25 June 2012. Available online at https://www.opennetworking.org/wp-content/uploads/2014/10/openflow-spec-v1.3.0.pdf

37. Ferrús R. (Editor). D4.5 Final network resource management framework and performance assessment of algorithmic solutions. H2020 VITAL project. August 2017. Available online at http://www.ict-vital.eu/documents/deliverables

38. AMARI LTE 100. https://www.amarisoft.com. Last accessed 13 October 2018.

39. OpenSAND. http://opensand.org. Last accessed 15 October 2018.

40. Ryu. https://osrg.github.io/ryu/index.html. Last accessed 15 October 2018.

41. Northbound Networks. Zodiac FX Product. https://northboundnetworks.com/collections/zodiac-fx. Last accessed 15 October.

42. Miller C. How and why commercial high-capacity satellites offer superior performance and survivability in the future space threat continuum. 32nd Space Symposium, Technical Track. April 11-12, 2016. Colorado Springs, Colorado, USA.

43. Whitepaper. Why Latency Matters to Mobile BackhaulÂă-ÂăO3b Networks. O3b Networks. 2013.

44. Iperf. The ultimate speed test tool for TCP, UDP and SCTP. https://iperf.fr/. Last accessed 18 October 2018. 